\title{
CIENCIA, EDUCACIÓN Y FORMACIÓN DE VALORES. PAPEL DE LA UNIVERSIDAD EN LA ACTUALIDAD.
}

\section{SCIENCE, EDUCATION AND VALUES, ROLE OF THE UNIVERSITY.}

\section{Margarita León García (PhD)}

Universidad de Ciencias Pedagógicas "Héctor Alfredo Pineda Zaldívar". Calzada de Arday y calle 100. Reparto El Trigal. La Habana, Cuba

Universidad Laica Vicente Rocafuerte de Guayaquil. Ave. Las Américas. Guayaquil, Ecuador

e mail: mleong@ulvr.edu.ec

\section{RESUMEN}

La formación de valores en los jóvenes y, específicamente, el papel de la universidad en esta labor, es una necesidad imperiosa en los momentos actuales en que la sociedad se enfrenta cada vez más, a situaciones problemáticas que requieren de la toma de decisiones que involucran un comportamiento ético de las personas. El trabajo que se presenta, plantea las reflexiones de la autora acerca de la relación entre el desarrollo científico y el proceso educativo en general a la vez que incorpora argumentos que destaquen el papel que le corresponde a los investigadores en el progreso social, así como sus relaciones de interdependencia. La educación en su sentido amplio y la escuela en particular, tienen la alta responsabilidad de formar en sus egresados, elevados valores morales que les permitan involucrarse conscientemente en la construcción de la sociedad. Para su realización se ha utilizado básicamente la revisión de la literatura especializada y los métodos teóricos de investigación, entre ellos, el método histórico y lógico, el enfoque de sistema y el análisis, la síntesis, la inducción, la deducción y la generalización.

Palabras clave: ciencia, educación, formación de valores, papel de las universidades en la formación de valores.

\begin{abstract}
Values-based education for young people and specifically the role of the University, is an absolute necessity nowadays when society faces increasingly problematic situations that require decision-making that involves an ethical behavior of people. The research presents the reflections of the author about the relationship between scientific development and the educational process in general while incorporating arguments that highlight the role of researchers in social progress, as well as their interdependent relationships. In its broadest sense, education and school in particular, have the big responsibility of teaching high moral values to their graduates so that they can consciously engage in the construction of society. Revision of specialized literature and theoretical methods of research have been made, such as the historical and logical method, systems approach, analysis, synthesis, induction, deduction, and generalization.
\end{abstract}

Keywords: science, education, values education, role of universities in values-based education

Recibido: octubre de 2014 Aprobado: noviembre de 2014

\begin{abstract}
"Un hombre puede saber más que otro hombre, puede saber bastante más, mucho más; pero si ambos han sido educados de modo que sepan desarrollar todas las fuerzas virtuales que poseen en calidad de hombres, la sociedad les ha dado cuanto está obligado a darles, y ellos a su vez en amplitud de resarcir plenamente lo que de la sociedad han recibido. El sabio trabajará como sabio, el industrial como industrial, el obrero como obrero, pero todos en pleno desenvolvimiento de su capacidad y de su energía física y moral. La educación completa es la que forma hombres completos".
\end{abstract}




\section{Introducción}

Es muy propio de las generaciones adultas, observar con atención la conducta de las nuevas generaciones, y tratar de determinar en qué se parecen a ellos, en qué se diferencian, qué traen ellas al mundo en términos de valores, conductas, modos de actuar. $Y$ esto es muy lógico, pues la juventud de hoy será el mañana de la sociedad y de acuerdo a cómo veamos que esta se comporta hoy, así pensamos cómo será el futuro.

Al leer el libro "La Educación en Secundaria" del autor A. Múdrik, se pueden encontrar las siguientes opiniones acerca de la juventud: (2)

"A nuestra juventud le gusta el lujo, está mal educada, se burla de la superioridad y no respeta en absoluto a los ancianos. Nuestros hijos de hoy se han convertido en tiranos, no se ponen de pie cuando en una estancia entra una persona de edad, contradicen a sus padres. Hablando en plata, son muy malos.

He perdido toda esperanza en cuanto al futuro de nuestro país, si la juventud de hoy empuña mañana las riendas del poder. Pues esta juventud es insoportable, impulsiva, simplemente horrible.

El mundo en que vivimos ha alcanzado una fase crítica. Los hijos ya no obedecen a sus padres. Por lo visto, el fin del mundo no está ya muy lejos.

Esta juventud está podrida hasta el fondo de su alma. Los jóvenes son pérfidos y negligentes. Nunca se parecerán a los jóvenes de otros tiempos. La joven generación de hoy día no sabrá conservar nuestra cultura".

Opiniones como estas las hemos escuchado en boca de abuelos, padres, maestros y adultos en general, son muy contemporáneas. Sin embargo, en el propio libro se citan de qué épocas son estas opiniones: (Mudrik, A. 1983)

La primera opinión pertenece a Sócrates que vivió entre los años 470399 a.n.e.; la segunda a Hesíodo, poeta griego, cerca de 720 años a.n.e. El autor de la tercera opinión es un sacerdote egipcio que vivió más de 2000 años antes de nuestra era. Y la cuarta acusación, se halló en una vasija de barro encontrada entre las ruinas de Babilonia y que data de más de 3000 años a.n.e.

Evidentemente, resulta incomprensible que si los jóvenes de esos tiempos eran tan malos, la humanidad y la civilización hayan podido sobrevivir y lograr los enormes progresos sociales a los que asistimos hoy, testimonio de los cuales somos nosotros mismos. Ocurre que en todos los tiempos, la juventud no ha sido homogénea y junto a su parte de avanzada, hay siempre en ella otros, cuya conducta no siempre es positiva y es advertida por los que la rodean.

Pero la conclusión más importante que se puede extraer de lo anteriormente expuesto es, a nuestro juicio, otra: en todos los tiempos ha habido preocupación de los adultos por la formación de los jóvenes y porque su educación responda a los más altos intereses de la sociedad, porque sus valores y forma de comportarse en ella, sean socialmente aceptados y por lo tanto de valor social.

Reflexionar acerca de la formación de valores en los jóvenes y específicamente del papel de la universidad en esta labor es el propósito de este trabajo, en el cual además, intentaremos relacionar este proceso con el fenómeno educativo en general y el desarrollo de la ciencia, expresar algunos argumentos que destaquen el papel que cada uno de ellos desempeña en el progreso social, así como en sus relaciones de interdependencia.

El trabajo que se presenta es el resultado de la consulta bibliográfica en diversas fuentes relacionadas con la temática y de reflexiones personales de la autora a partir de su experiencia de trabajo y de vida.

\section{Desarrollo}

\section{Ciencia desarrollo social sur- gimiento de los valores}

El desarrollo de la ciencia y su progreso están determinados por las demandas de la sociedad, es decir, su desenvolvimiento no puede analizarse si no es en la concatenación con la práctica productiva social. Sin tratar de analizar el desarrollo de la ciencia, lo que sería muy amplio y complejo, resulta necesario recordar hitos fundamentales de dicho desarrollo.

El surgimiento de la ciencia se ubica en la Grecia Antigua, después de la religión, la moral y el arte. Surge como respuesta a las demandas de la producción. En esta etapa inicial, la ciencia evolucionó muy lentamente, debido al predominio de la fragmentación política, luego al despotismo monárquico y a la influencia que ejerció la religión sobre todas las formas de la conciencia social, lo que provocó, un estancamiento de la ciencia.

El surgimiento de la burguesía y el nuevo modo de producción capitalista, propiciaron un impulso al desarrollo de las modernas ciencias naturales 
experimentales, se sistematizaron conocimientos sobre las disciplinas sociopolíticas y surge una nueva filosofía: la filosofía moderna. Este proceso en ascenso tiene un punto culminante en la Revolución Industrial en la cual, la interrelación ciencia técnica se expresó en el papel determinante de la producción y por lo tanto, de la técnica, y se impulsaron considerablemente las ciencias sociopolíticas y la filosofía.

Esta evolución continuó y originó un salto cualitativo en la relación ciencia técnica y el desarrollo científico, que es la fase actual conocida como Revolución Científico Técnica. Ella ha transitado por distintas fases de desarrollo: una fase en la cual la estrategia era desarrollar la ciencia para desarrollar la tecnología y así obtener mayores riquezas; esta fase dio paso a una superior, desarrollar ciencia y tecnología unidas para obtener mayores riquezas. En la más cercana actualidad se habla de desarrollar estrategias de innovación, es decir, la ciencia en función de la innovación tecnológica y que ello se revierta en hacer cada vez mejor lo que a cada cual le toca hacer.

Es decir, la ciencia, que inicialmente fue impulsada por las necesidades de la producción, ha pasado a ser un punto de partida para revolucionar e innovar la práctica. En opinión de García, G.J. (3), el progreso científico está condicionado por diversos factores objetivos y reales y señala que ellos son el desarrollo de la propia práctica, las demandas de la producción, la dirección planificada de la sociedad, las necesidades militares, la lucha de clases, la protección de la salud, la protección de la naturaleza y la educación de las nuevas generaciones.

Observemos como entre esos factores se señala la educación, que no por estar en último lugar resulta menos importante. Más adelante veremos que la educación juega un papel destacado en el progreso social y por lo tanto en el desarrollo científico.

La ciencia contemporánea ha dejado de ser cada vez más, una mera actividad académica, de gabinete, que se lleva a cabo en los límites estrechos de un laboratorio o una institución especializada, para convertirse en fuerza social activa que influye de manera determinante en los aspectos más diversos de la sociedad, la producción, el modo de vida y la cultura en general. La ciencia no es un elemento de instrucción e ilustración, ni siquiera solo una riqueza teórica sino, también, una riqueza práctica de la sociedad. En la ciencia como fuerza social activa se funden de manera especial el pensamiento y la acción.
La ciencia tiene su base y fundamento, su fuerza propulsora, en la actividad práctico material de los hombres, en las necesidades de la producción social, encaminadas a la transformación de la realidad. A partir de la práctica, la ciencia constituye, a su vez, un aspecto necesario de esta actividad material transformadora, un instrumento esencial de la sociedad, concebida como sistema en autorregulación y en autodesarrollo. Esto da lugar a que la ciencia se torne fuerza productiva directa.

De hecho, la ciencia amplía de modo considerable la esfera de su acción y revoluciona profundamente las bases del proceso social, pero el valor práctico de la ciencia se expresa también, en la esfera de los conocimientos sociales y humanísticos. El conocimiento científico se convierte en un instrumento teórico de comprensión y transformación social al esclarecer las bases y vías de los cambios y transformaciones sociales.

La ciencia como fuerza social integral, permite una utilización plenamente científica de todos los resultados y logros, es decir, una utilización que responda a cabalidad a los intereses y valores esenciales del individuo y de toda la humanidad. Considerar la ciencia como una actividad social determinada conduce a su enfoque valorativo, a incluirla en la órbita del análisis axiológico.

El problema de la relación de la ciencia y el valor tiene dos aspectos fundamentales:

- El valor de la ciencia en relación con la sociedad, aspecto al que nos hemos referido en los párrafos anteriores $y$,

- La presencia de aspectos valorativos en la propia ciencia.

Este segundo aspecto no es explicado igual por las distintas corrientes de pensamiento filosófico. El pensamiento positivista burgués absolutizó la problemática del valor a partir de concepciones idealistas objetivas, idealistas subjetivas o pragmático naturalistas que tienden a reducir los valores a una esfera trascendente del deber ser, a una región puramente normativa del carácter subjetivo, cerrando con ello las puertas al análisis científico de la naturaleza verdadera del valor y de las relaciones entre el valor y la ciencia. La tendencia fue establecer una dicotomía entre hecho y valor, entre lo descriptivo y lo valorativo, entre el ser y el deber ser, entre la realidad y el ideal.

Esta comprensión de la ciencia como fenómeno axiológicamente neutral, implica considerar que la ciencia no representa un valor real, aunque de ella se obtengan beneficios prácticos. Las corrientes neopositivistas actuales establecen una imcompatibilidad aún mayor entre ciencia y valor: 
si la ciencia constata hechos y por eso es verificable, y los valores expresan la relación subjetiva del hombre con la realidad y por lo tanto no son verificables, entonces las proposiciones valorativas, al no ser ni verdaderas ni falsas, ni demostrables ni refutables, carecen de sentido en el plano científico.

La filosofía materialista dialéctica establece la necesidad del análisis objetivo de los valores, a partir del principio del determinismo aplicado a la vida social. Es cierto que es posible diferenciar formas del pensamiento científico y formas del pensamiento valorativo, pero también se establece un nexo interno, tránsitos recíprocos e interacciones entre ciencia y valor. En este sentido, el enfoque dialéctico permite transformar el valor en conocimiento, con lo que se amplían las posibilidades cognoscitivas del hombre y el valor de la ciencia reside en que en la medida en que el conocimiento penetra en la vida se hacen más amplias y plenas la libertad y la creación humanas como valores permanentes del hombre.

La relación valorativa constituye un lado de la relación práctica del sujeto, derivada de ella. A través de la relación valorativa, el hombre representa el mundo, en correspondencia con sus necesidades y ello está vinculado a la proyección ideal de los objetivos y fines que persigue. Esta relación valorativa se da en todas las esferas de la actividad humana y por lo tanto, es inherente también a las ciencias.

Su importancia radica en que revela el mecanismo de interacción entre la actividad práctica y la actividad cognoscitiva. El hombre valora para hacer más efectiva su práctica y a la vez los valores guían la actividad humana en todas sus manifestaciones.

Llegado a este punto, es necesario entonces, referirse al papel que juega el hombre que hace ciencia. El investigador, aunque no lo exprese, no quiera o no se proponga expresarlo, es un hombre no liberado de ninguna de las responsabilidades del hombre en su dimensión histórico concreta. No es un sujeto desideologizado ni amoral, ni puramente neutral. Tiene el deber pleno y no compartido, de no ceder a otros el juicio de valor implícito en la naturaleza de la finalidad teórica y práctica del quehacer científico.

El investigador es portador de determinada orientación valorativa dentro de la propia ciencia la cual guía la elección de parámetros lógico metodológicos sobre cuya base se valoran y escogen las formas y modos de descripción, explicación, demostración y organización del conocimiento. El científico es quien por lo general, emite la primera valoración sobre la significación metodológica y social en los resultados obtenidos en la investigación científica.

En la formación de los futuros investigadores, hombres dedicados a la ciencia, igual que en la formación de todo hombre, tienen que estar presentes los valores y esto es responsabilidad primaria de la educación como fenómeno social que tiene su concreción pedagógica en el proceso educativo que se desarrolla en la escuela, desde la educación inicial hasta la educación superior.

\section{La educación la escuela la for- mación de los valores}

Siguiendo el criterio de Hernández, I. (4), la educación tiene dos funciones generales y fundamentales: función formativa y función desarrolladora. Formando parte de la formativa está potenciar el desarrollo social, formar valores, actitudes, normas de conducta, desarrollar y enriquecer cultural, emocional y sentimentalmente, entre otras. En las desarrolladoras está la de formar la fuerza laboral científica, profesional e intelectual.

Existe una relación estrecha entre la formación del hombre y la educación como formadora de valores. La formación de valores en un hecho histórico ligado a la evolución del propio hombre. Tanto lo que se educa, como lo que se educa, reflejan el adelanto de la conciencia de la humanidad y suponen resultado de una acumulación.

Desde su surgimiento, la escuela ha asumido la responsabilidad de la formación de valores. La Escuela como institución dedicada a la educación y a la enseñanza, surgió en la Antigua Grecia, alrededor de los años 600 a.n.e. La misma vino a desempeñar una función que ya no podía seguir realizando ni la tradición oral ni la imitación de los adultos, que era la enseñanza de las "letras". Aunque en su mayoría eran escuelas particulares (estas que surgieron inicialmente), el Estado reglamentaba el tipo de educación que el niño debía recibir en la familia y en las escuelas.

El maestro debía formar a los futuros gobernantes e inculcarles el amor a la patria, a las instituciones y a los dioses. Es decir, desde su surgimiento, la escuela fue encargada de transmitir los valores predominantes de la época y de la clase social en el poder y hacer que ellos se asimilaran por los jóvenes y se tradujeran en conductas.

Pudiéramos citar algunos ejemplos que ilustren lo planteado en los párrafos anteriores. En la antigua civilización india, se enseñaba esencialmente 
a través de proverbios y fábulas que contenían preceptos morales. En la escuela elemental, se aprendía de memoria el catecismo budista que prescribía: (5)

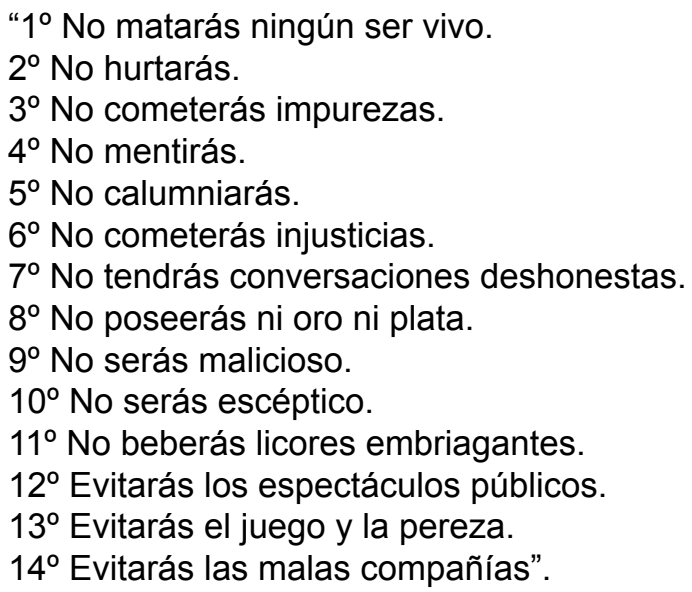

Como se puede observar, el aprendizaje memorístico de estos mandamientos de "obligatorio cumplimiento" (las comillas son de la autora) contenían los valores predominantes en la antigua sociedad india.

Otro ejemplo es Esparta, en Grecia Antigua, donde se afirma que en la escuela elemental "... la educación específicamente moral, no fue nunca descuidada. El respeto a los ancianos, la obediencia, el sentimiento del honor, fueron virtudes comunes a los espartanos" (6)

Al evolucionar la sociedad y las ideas acerca de la educación, surgen las opiniones pedagógicas, las teorías sobre la educación. En la teoría de la educación se resume el ideal pedagógico de una clase social y allí están los valores que deben ser transmitidos. Ese ideal pedagógico se traslada a la escuela y en él se expresa de un modo parcial o acabado la concepción del mundo imperante en cada época.

Así ha ocurrido en el devenir histórico de la humanidad hacia sociedades cada vez más avanzadas. A la escuela le ha correspondido esa función: transmitir y lograr la apropiación de la experiencia histórico social anterior. Los más grandes educadores de la humanidad, han señalado la importancia de la escuela como centro responsabilizado con la función educativa, lo que incluye junto a otros aspectos, la formación de los valores.

José de la Luz y Caballero, eminente maestro cubano, se reunía en las tardes en el Colegio El Salvador con los niños, los maestros e incluía personas ajenas a la escuela y leía un párrafo de un libro, no importaba de qué asunto tratara, pero siempre era válido para hablar del deber, la res- ponsabilidad, del sentido moderno de la justicia, de la moral y este era el centro de sus preocupaciones educativas.

En sus Elencos y Discursos Académicos, aparece lo siguiente: "Así como el pintor, el poeta y el escultor hacen nacer en medio de una inspiración divina multitud de seres que eternamente veremos moverse y agitarse porque se hallan dotados de una verdadera vida, así también, el educador crea nuevos seres, los hace hombres, les infunde la vida del espíritu y les inspira los invariados y verdaderos principios de su existencia, principios que han de ser tanto más grandes cuanto que conforme a ellos han de realizarse muchas esperanzas allí cifradas por su patria y su familia...." (7)

José Martí, el Héroe Nacional de Cuba, expresó en sus escritos el concepto, funciones y carácter que para él tenía la escuela y además de exponer su función instructiva, también se refirió a la transmisión de valores y a la relación entre la escuela y la sociedad que la crea y la sostiene. Así señaló: "El pueblo más feliz es el que tenga mejor educados a sus hijos, en la instrucción del pensamiento y en la dirección de los sentimientos". (8)

Centremos la atención en el papel de la escuela en la formación de los valores. La escuela que, obviamente, no puede abstraerse del contexto social en el cual ella se encuentra, que no está sumergida en una urna de cristal que la protege de las influencias del medio; que todos sus miembros: estudiantes, docentes, personal de apoyo y directivos, viven en una sociedad donde están presentes todas las contradicciones que caracterizan nuestro mundo de hoy.

Antes precisemos, qué son los valores y a cuáles nos referimos cuando hablamos del papel de la escuela en su formación.

En el Diccionario Filosófico (1984) se definen los valores como: "Determinaciones sociales del mundo circundante, que ponen de manifiesto su significación positiva o negativa para el hombre y la sociedad.(...) Exteriormente los valores constituyen las propiedades del objeto o fenómeno, pero no le están dados por la naturaleza, no le son innatos en virtud de la estructura interna del objeto por sí mismo, sino porque este último está incorporado a la esfera del ser social del hombre y se ha convertido en vehículo de las relaciones sociales concretas..." (9)

Miranda, T. (1998) define los valores como: "Proyectos ideales que surgen sobre la base de las necesidades y actividad humana y contienen anticipaciones acerca de qué es lo que hay que 
transformar en la realidad y en el propio hombre. Son los fines que orientan la actividad humana y el conocimiento". (10)

En ambas definiciones hay cuestiones comunes y esclarecededoras acerca de los valores: no existe valor de objeto o fenómeno alejado de la significación que este tiene para las personas y el valor de los objetos y fenómenos está dado por la relación conocimientos actividad práctica. Los valores en tanto, parte constitutiva de la propia realidad social, desempeñan una determinada función en la sociedad, adquieren una u otra significación social, favorecen u obstaculizan el desarrollo progresivo de la sociedad. (11)

Se trata entonces de formar valores. Por formación se entiende, en coincidencia con Bermúdez, R. (2011), el "proceso de interacción entre educadores y estudiantes, en condiciones de actividad y comunicación que faciliten la apropiación de la experiencia histórico social y el crecimiento personal de todos los implicados". (12)

Cuando hablamos del papel de la escuela en la formación de los valores, generalmente nos referimos a la educación moral, cuestión que implica, una fragmentación de la educación, pues toda educación es moral. Es imposible el acto de educar fuera de su sentido moral, pero no siempre se explicita la intencionalidad moral en la educación lo que hace que se conciba como algo específico e independiente de lo que ocurre en el proceso educativo integral.

La moral abarca todas las formas y mecanismos de nuestras relaciones con los otros. Su contenido tiene como función esencial el desarrollo de valores que orienten de la forma más justa y humana las relaciones entre las personas. Sin embargo, aquí ya comienza la complejidad del asunto de la formación y desarrollo de los valores, pues ocurre que para todas las personas no es lo mismo lo justo y lo humano. $Y$ es que los valores por su naturaleza constituyen la unidad de lo objetivo y lo subjetivo y surgen solo en la relación sujeto objeto. Esta relación es cognoscitiva y práctica, ambas están estrechamente vinculadas, se condicionan y penetran mutuamente,

Según González, F. (1998), el problema de la formación de los valores se enfrenta a, por lo menos, dos cuestiones básicas: el sentido que adquieren los valores morales para la persona, y la forma en que intencionalmente asume y desarrolla los valores. (13)

Es decir, los valores no tienen un sentido estático. Su contenido no define un sentido absoluto fuera de su personalización por un sujeto concreto, en otras palabras, es imposible buscar el valor real de un contenido moral fuera del sentido subjetivo que tiene para la persona que debe asumirlo.

Por lo tanto, la esencia de la educación de los valores no es la transmisión de contenidos y valores establecidos sino, el proceso de configuración conjunta con el estudiante de un sistema de valores que él pueda personalizar, porque tienen un sentido moral para él y que sea realmente vivenciado y asumido.

La educación de los valores tiene un sentido orientado por la sociedad. Se trata de convertir los valores sociales en valores individuales para las personas. Esto tiene que ser un proceso en el cual el estudiante participe y que él sea sujeto de su propia formación. Por ello, la formación de los valores no puede sufrir del dogmatismo que a veces lastra nuestro quehacer educativo, pues el dogmatismo presupone la no individualización de la expresión moral, exigiendo una total homogeneidad en las conductas y sus formas individuales de expresión.

De acuerdo con lo planteado por el citado autor, es posible resumir un grupo de requerimientos psicopedagógicos para la formación de los valores, que deben ser tenidos en cuenta en la labor pedagógica por los docentes. Ellos son:

- Toda actividad dirigida a la formación y desarrollo de valores en la escuela debe partir del vínculo con la experiencia y las necesidades reales, pues el estudiante incorpora y da sentido a aquello que se vincula con sus experiencias y necesidades.

- Aprovechar al máximo las posibilidades que da la comunicación docente-estudiante, pues esta puede ampliar en el alumno su sensibilidad para hacer suyos nuevos aspectos de la vida que si bien de inicio no adquieren sentido para él, lo adquieren a través de la comunicación.

- La personalización de los valores implica la congruencia de los nuevos valores que se desean educar con una racionalidad asumida individualmente. No se pueden imponer los valores.

- La comunicación interpersonal entre los estudiantes favorece la formación de los valores por la calidad afectiva que le imprime al proceso, que incluye emociones vivenciadas colectivamente que ayudan al desarrollo de la sensibilidad del estudiante hacia diferentes as- 
pectos de la vida.

- El proceso de formar valores no es independiente de la formación y desarrollo de otras configuraciones psicológicas como son los ideales morales, las necesidades, las motivaciones, las intenciones, la concepción del mundo, por lo tanto hay que hacerlo en estrecha interrelación.

En el caso particular de las universidades, la formación de los valores está muy ligado a la actividad laboral, es decir, la formación de conciencia y conductas morales, estéticas, económicas, político ideológicas, vinculadas a la actividad de trabajo.

El objetivo esencial de las universidades es la formación de profesionales competentes, que junto a las competencias dirigidas a la utilización de recursos, destrezas interpersonales para el trabajo en equipos, información adquisición y evaluación de datos, tecnología selección de equipos e instrumentos y de competencias fundamentales para la lectura, redacción, expresión, capacidad de escuchar, analizar de modo creativo, tomar decisiones, solucionar problemas, saber razonar y aprender por sí mismos; unan cualidades personales que reflejen valores tales como la responsabilidad, laboriosidad, honestidad, honradez, patriotismo, colaboración mutua

Ello solo es posible si se educan intencionadamente los valores en cada una de las actividades del proceso pedagógico profesional. En este sentido es necesario lograr en los estudiantes universitarios, varios objetivos específicos. Entre ellos están: (14)

1. La educación de la conciencia de la responsabilidad social en el trabajo y hacia toda actividad socialmente útil, lo cual implica la formación del estudiante en la necesidad y utilidad de su trabajo o profesión, el desarrollo de la satisfacción y orgullo profesional por la labor que realiza, la adhesión a la profesión, la conciencia de iniciativa y responsabilidad, la disposición hacia el rendimiento y desarrollo en general de una atmósfera de optimismo en el colectivo laboral.

2. La educación de la disposición laboral, es decir la obtención de altos rendimientos y elevación de la calidad laboral para obtener mayores logros productivos, la capacidad para cambiar de puesto de trabajo y desempeñarse con éxito y para emprender.

3. La educación de la creatividad e iniciativa, que implica desarrollar imaginación para solucionar problemas. Aquí los valores que el estudiante haya desarrollado tienen un papel decisivo pues la creatividad es ante todo una cuestión de poder, pero la iniciativa es una cuestión de querer. Ello se relaciona notablemente con el desarrollo de la disposición y de la independencia.

4. La educación de la disciplina laboral, que se relaciona con el desarrollo de cualidades dirigidas al cumplimiento en su trabajo, con exactitud y de la mejor manera, la observación de las informaciones y orientaciones sobre el proceso productivo, el cumplimiento de las normas de calidad, el mantenimiento de la disciplina tecnológica, la conducta disciplinada en el colectivo y con la dirección, todo lo cual expresa el desarrollo de valores como honestidad y responsabilidad.

5. La educación del colectivismo y la adhesión al oficio y al centro de trabajo. Este es básico para la futura inserción a un colectivo laboral y la forma en que asimile la influencia de dicho colectivo para lograr el desarrollo de conciencia, permanente ejecución profesional con alto rendimiento, disposición positiva hacia las exigencias laborales cambiantes en todas las profesiones.

Es una necesidad insoslayable en el mundo actual que el futuro profesional desarrolle todos estas cualidades a través de las cuales se expresan los valores, para poder enfrentarse con éxito al trabajo. $Y$ estos desafíos que impone el mundo del trabajo en la actualidad, impone retos que debe asumir el sistema educativo para formar los trabajadores y profesionales necesarios para participar activamente en los nuevos sistemas productivos.

\section{Conclusiones}

El problema complejo y desafiante de la formación y desarrollo de los valores es un asunto a enfrentar por diversos factores sociales, cada uno con responsabilidades concretas, pero indiscutiblemente que una de las principales responsabilidades le corresponde a la escuela, por tradición y por preparación para enfrentarlo.

En la formación y desarrollo de los valores se resumen varios problemas sociales de la ciencia en la actualidad: la relación entre lo objetivo y lo subjetivo, la necesidad del enfoque axiológico de la ciencia, la actividad científica y los valores, los científicos y los juicios de valor, la necesidad de desarrollar una conciencia moral y humanista de la actividad científica, el hombre como sujeto y objeto de la ciencia, entre otros.

Las universidades tienen que repensar sus estrategias de trabajo para la formación de los valores, alejada de dogmas y doctrinas, acercando más al 
estudiante a la realidad con la cual se enfrentará. Buscar formas novedosas a través de las cuales educar los valores a los jóvenes que serán los trabajadores y científicos del mañana, a los que hay que educar de modo que puedan desarrollar su vida y trabajo en la sociedad actual, integrada a un mundo heterogéneo y cambiante.

\section{Referencias}

(1) Varona, E. J. Trabajos sobre educación y enseñanza. La Habana. Editorial Pueblo y Educación, (1992). p. 200.

(2) Múdrik, A. La educación en Secundaria. Moscú. Editorial Progreso, (1983). pp. 8-9.

(3) García, G. J. Ciencia, Filosofía e Ideología. La Habana, Cuba. Editorial Científico-Técnica. (1985).

(4) Hernández, I. Acerca de la Formación de Valores y la Responsabilidad Ciudadana. (Ponencia). La Habana, Cuba. Universidad de Ciencias Pedagógicas "Héctor A. Pineda Zaldívar". (2012).

(5) Larroyo, F. Historia General de la Pedagogía. México. Editorial Porrúa, S.A, pp. 75-76.

(6) Idem, (1984). p. 135.

(7) Luz, J. de la. Dos discursos leídos en el Colegio El Salvador. En Obras Tomo III. P. 482. Recuperado de: www.books.google.com.ec/ books?isbn $=849007786 X$. (1861) Consultado en noviembre de 2014.

(8) Martí, J. Ideario Pedagógico. La Habana, Cuba. Editorial Pueblo y Educación, (2007) p. 147.

(9) Diccionario de Filosofía. Moscú. Editorial Progreso, (1984). p. 439.

(10) Miranda, Teresa. Conferencia Magistral. Evento Base Pedagogía '99 del ISPETP. La Habana, 26 de junio de 1998. (1998). (Notas tomadas por la autora).

(11) Fabelo, J. R. Los valores y sus desafíos actuales. Colección Insumisos latinoamericano. Recuperado de: www.lirosenred.com. (2004). Consultado en octubre 2014.

(12) Bermúdez, R. La categoría formación. La Habana, Cuba. Universidad de Ciencias Pedagógicas "Héctor A. Pineda Zaldívar", (2011). p.3. (En soporte digital)

(13) González, F. Los valores y su significación en el desarrollo de la persona. La Habana, Cuba. Revista Temas 15. (1998).

(14) Abreu, R. Pedagogia Profissional: uma proposta aberta à reflexão e ao debate. São Paulo, Brasil. UCPETP- CETEC Paula Souza.Brasil. (2013). 